\title{
Repair of the anophthalmic cavity of rats with synthetic hydroxyapatite
}

\author{
J.J. Ranzani', \\ S.C. Rahal ${ }^{1}$, \\ S.A. Schellini ${ }^{2}$, \\ M.E.A. Marques ${ }^{3}$ \\ and E.M. Taga ${ }^{4}$
}

\author{
'Departamento de Cirurgia Veterinária e Reprodução Animal, \\ Faculdade de Medicina Veterinária e Zootecnia, \\ Universidade Estadual Paulista, Botucatu, SP, Brasil \\ Departamentos de ${ }^{2}$ Oftalmologia e Otorrinolaringologia and ${ }^{3}$ Patologia, \\ Faculdade de Medicina, Universidade Estadual Paulista, Botucatu, SP, Brasil \\ ${ }^{4}$ Departamento de Bioquímica, Faculdade de Odontologia, \\ Universidade de São Paulo, Bauru, SP, Brasil
}

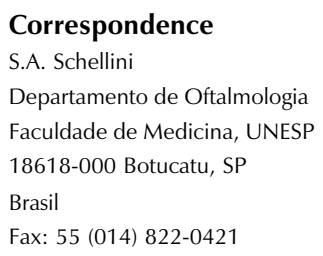

\begin{abstract}
We placed spheres of synthetic hydroxyapatite (calcium chloride combined with sodium phosphate) in the eviscerated or enucleated orbital cavity of rats in order to evaluate the biocompatibility of this material with the orbital cavity. The study was conducted on 50 albino rats, 25 of which were submitted to enucleation and 25 to evisceration of one eye. The animals were sacrificed 7, 15, 21, 30 and 60 days after surgery and the orbital content was submitted to histopathological examination. A reaction of the young granulation tissue type was observed first. The hydroxyapatite was gradually surrounded by a granulomatous macrophage inflammatory response and covered with dense connective tissue that formed a sort of "mesh" septating and supporting progressively smaller blocks of the substance. The same type of reaction was observed in the enucleated and eviscerated cavities. We conclude that synthetic hydroxyapatite is an inert nonallergenic material which is appropriate for volume replacement in the anophthalmic cavity.
\end{abstract}

\section{Introduction}

The loss of the ocular globe or of its content may lead to orbital asymmetry and even to facial asymmetry if the lost orbital volume is not replaced. Volume replacement has been performed for the past 100 years, starting with Mules who developed glass spheres (1). After World War II, new materials were used to manufacture the spheres, the most diffuse one being silicone, which was developed in the U.S. and widely employed all over the world.

The first spheres were manufactured with smooth-surfaced material in an attempt to
Key words

- Synthetic hydroxyapatite

- Reconstruction of the

anophthalmic cavity

- Experimental rats prevent the inflammatory response that might cause extrusion of the prosthesis from the cavity. However, the field of orbital implants was enriched by the studies of Perry (2) who suggested the use of natural hydroxyapatite, a porous surface product, for the reconstruction of anophthalmic cavities. In this situation, the fibrovascular tissue of the host fills the pores of the sphere and promotes better implant mobility. The fibrovascular response also causes the implant to become part of the orbit by integrating it into orbital tissues and preventing implant extrusion and migration.

Natural hydroxyapatite has been long uti- 
lized in dentistry and maxillofacial surgery and has proved to be a biocompatible and nonallergenic material. However, its price prevents its large scale use and new materials must be developed for use in the reconstruction of anophthalmic cavities. Thus, by extrapolating once again the knowledge existing in the area of dentistry, we are currently testing the use of synthetic hydroxyapatite for orbital volume replacement (3). The material presented here is a synthetic hydroxyapatite developed by E.M. Taga (one of the authors) in powder form which becomes a pasty resin when mixed with physiological saline, glucose solution or blood.

In the present study we used powdered hydroxyapatite spheres in eviscerated or enucleated rat orbital cavities in order to test the biocompatibility of the material with the orbital cavity.

\section{Material and Methods}

The study was conducted on 50 albino rats from the Central Animal House of the Botucatu Campus, UNESP, 25 of which were submitted to enucleation and 25 to evisceration. The animals were anesthetized $i p$ with $30 \mathrm{mg} / \mathrm{kg}$ sodium pentobarbital and maintained in ventral decubitus. All surgical procedures were performed on the right eye using a surgical microscope (Carl Zeiss, Jena, Germany) at 10X magnification.

\section{Surgical techniques}

Evisceration. $180^{\circ}$ conjunctival and perilimbic scleral opening; removal of the ocular content with a curette and Q-tip; preparation of hydroxyapatite spheres by the addition of physiological saline to powdered hydroxyapatite; placement of the hydroxyapatite sphere inside the scleral cavity; simultaneous closure of conjunctiva and sclera with continuous single 8-0 silk sutures.

Enucleation. $360^{\circ}$ conjunctival opening; section of the extrinsic muscles and of the optic nerve; removal of the ocular globe; removal of the content of the ocular globe; filling the scleral cavity with the hydroxyapatite sphere; closure of the sclera with 8-0 silk sutures; placement of the sphere covered with the sclera inside the orbital cavity; conjunctival closure with continuous 6-0 silk sutures.

The animals were maintained in cages under continuous observation, with free access to water and ration. Five rats per group were sacrificed $7,15,21,30$ and 60 days after surgery, and the content of the orbital cavity was removed, immersed in $10 \%$ formalin for 3 days and processed for histopathology according to the routine procedures of the Department of Pathology, UNESP, Botucatu. Slides were stained with hematoxylin-eosin. The same pathologist (MEAM) evaluated histopathologically all cases without being aware of the group to which the animal belonged.

\section{Results}

The animals showed a good postoperative course, feeding normally and maintaining physical activity inside the cage.

During the placement of the synthetic hydroxyapatite sphere we observed difficulty in the maintenance of its physical structure since the sphere, when in contact with blood, lost its shape, with the consequent possible loss of some material during closure of the cavity.

Both the enucleated and eviscerated animals presented contraction of the operated cavity at the end of the experiment. The volume of the scleral sac was smaller in the enucleated animals, whereas the structures were better maintained in the eviscerated animals. No animal developed inflammation of the orbital cavity or extrusion of the implant.

Histopathological evaluation showed progressive fibrous thickening of cornea and sclera from seven to 60 days in all animals. 
Seven days: The histopathological reaction of enucleated animals was similar to that of eviscerated animals but the sclera better defined the cavity in the latter. The cornea and sclera presented edema and inflammation. The ocular cavity presented voluminous hydroxyapatite "masses" with large amounts of inflammatory cells, especially phagocytes (macrophages), and the presence of young granulation tissue (Figure1).

Fifteen days: Reduction in the amount of hydroxyapatite and a larger number of inflammatory cells were observed in enucleated animals. In some cases the material was observed in the cytoplasm of macrophages, with the presence of a giant cell reaction. Focal points of fibrosis and granulation tissue were observed close to the sclera. The reaction of eviscerated rats was similar to that of enucleated rats. In some animals the substance was observed in smaller fragments compared to the 7-day group, most of it inside phagocytic cells (Figure 2).

Twenty-one days: The amount of visible hydroxyapatite was smaller and fibrous tissue was increased in both enucleated and eviscerated animals.
Thirty days: Two animals presented filling of the cavity with dense, sclera-like fibrous tissue. The remaining animals showed a pattern similar to that observed after 15 and 21 days, i.e., the persistence of a reasonable amount of hydroxyapatite associated with the granulomatous macrophage reaction and an intense plasmocyte infiltrate (Figure 3).

Sixty days: The reaction of both enucleated and eviscerated animals was similar and the inflammatory pattern was granulomatous, epithelioid and dense. The substance underwent calcification. In some animals there was formation of a dense epithelioid or sarcoid granuloma surrounding small fragments of the substance (Figure 4).

\section{Discussion}

Research for the development of materials for the reconstitution of anophthalmic cavities was intensified after Perry (2) suggested the use of natural hydroxyapatite, an inert nonallergenic substance that integrates into tissues. Other materials have been suggested for use in anophthalmic cavities, such as polyethylene, which is also nonallergenic

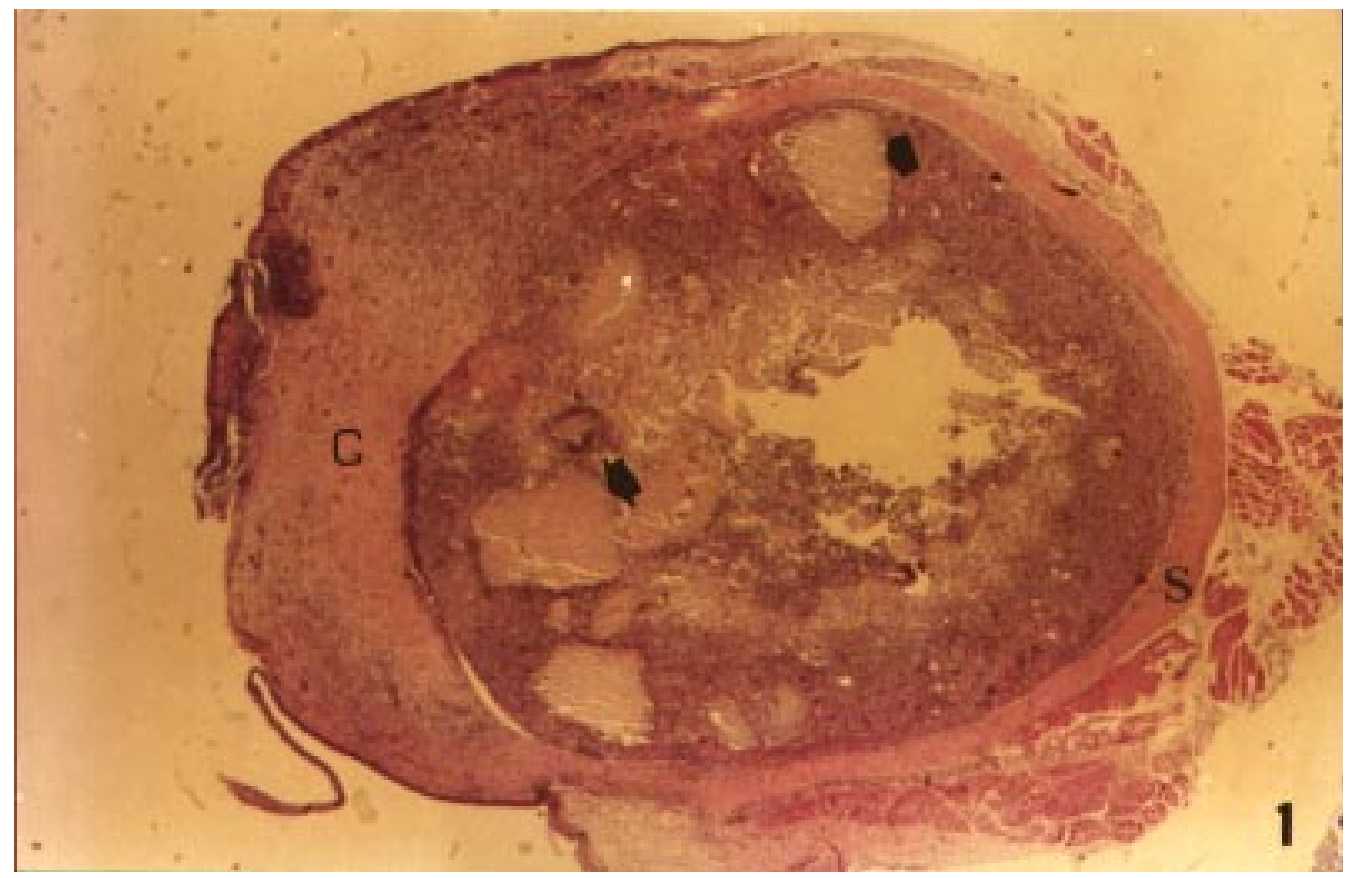

Figure 1 - Ocular cavity with "masses" of hydroxyapatite (arrows), young granulation tissue and inflammatory cells. S, Sclera; C, cornea (eviscerated 7-day group; HE, X40). 
Figure 2 - Hydroxyapatite (h) surrounded by young granulation tissue and inflammatory cells (enucleated 15-day group; HE, X100).

Figure 3 - Ocular cavity with fibrous tissue (f) and reduction of the amount of hydroxyapatite (arrow). S, Sclera; C, cornea (enucleated 30-day group; $\mathrm{HE}$, X40)

and permits growth of fibrovascular tissue in its pores (4).

However, due to the high cost of both natural hydroxyapatite and polyethylene, these materials are not routinely used and it is necessary to look for alternatives for the treatment of anophthalmic cavities. We suggest the use of synthetic hydroxyapatite consisting of calcium chloride combined with sodium phosphate. The present results show that the material is well tolerated by the cavity, with no rejection or extrusion occur-
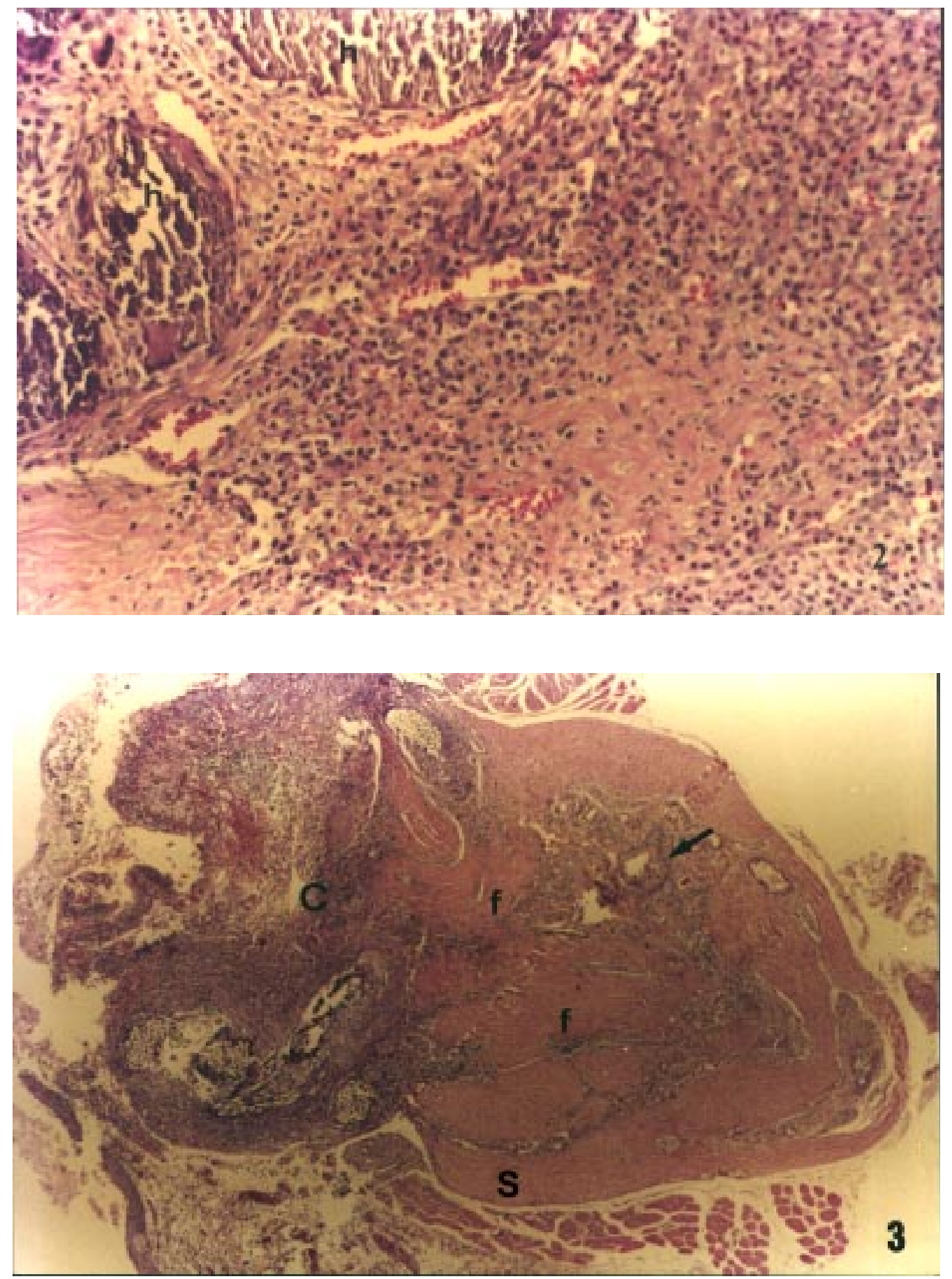


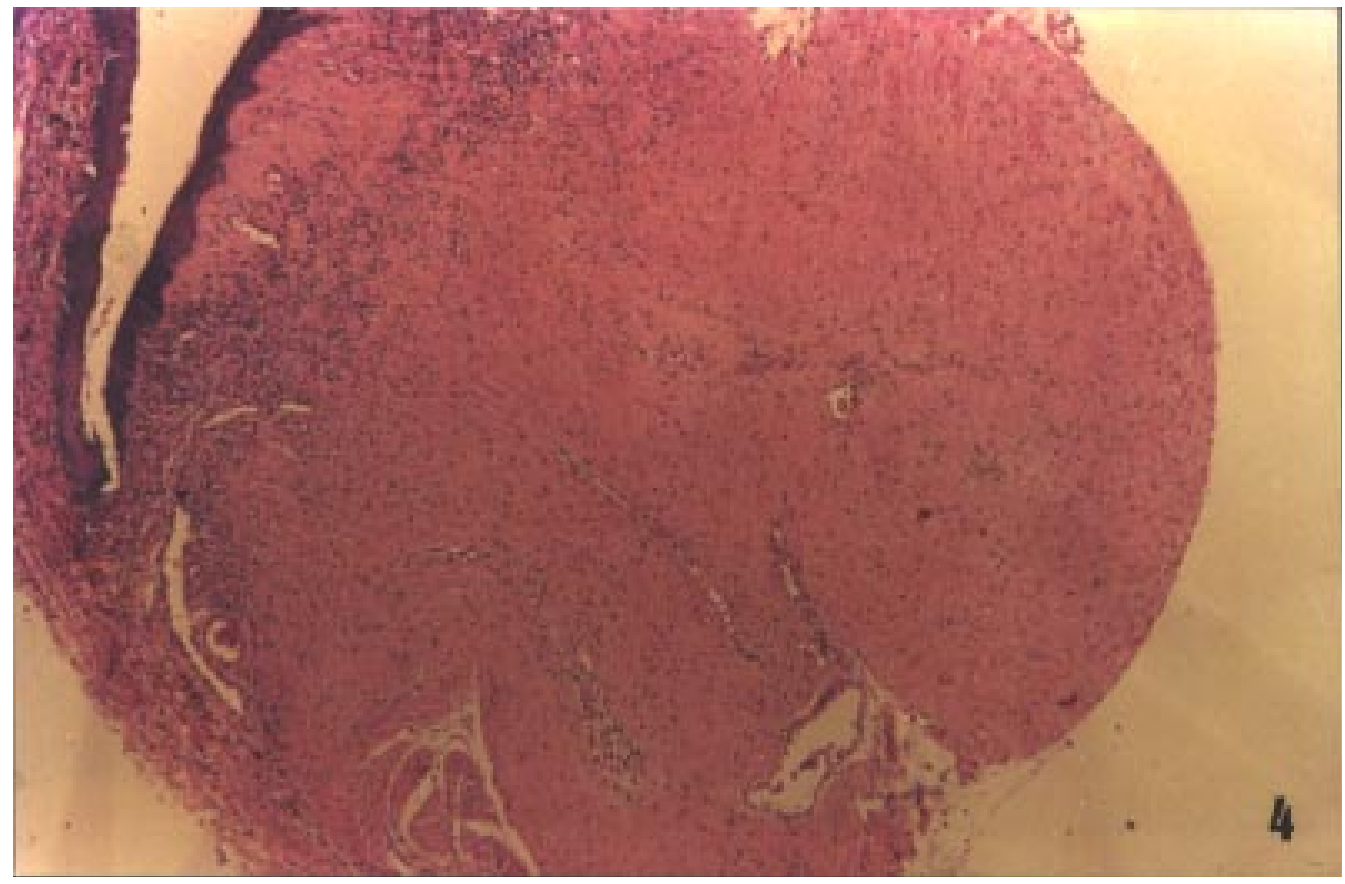

ring in any of the animals.

Enucleation and evisceration were performed in order to find out which of the surgical techniques would better adapt to the use of the substance tested. Because of the small dimension of the ocular globe of the rat, the technical difficulty was much greater when enucleation was used. With evisceration, the structure of the scleral sac is better preserved and it is well known that this procedure should be performed whenever possible, with enucleation being limited to cases of intraocular tumors, intense phthisis bulbi or traumatic injuries with extensive lacerations of the cornea/sclera.

Histopathological analysis demonstrated that the material is first surrounded by inflammatory cells that gradually phagocytize hydroxyapatite and replace it with connective tissue, which is initially weaker and later denser, with fibrosis. The same type of
Figure 4 - Dense fibrous tissue filling the entire ocular cavity (eviscerated 60-day group; HE, $\mathrm{X} 40$ ). 


\section{References}

1. Tonkelaar J, Henkes HE \& Leersun GK (1991). A short history of the artificial eye. Documenta Ophthalmologica, 77: 349354.

2. Perry AC (1990). Integrated orbital implants. Advances in Ophthalmic, Plastic, and Reconstructive Surgery, 8: 75-81.
3. Granjeiro JM, Taga EM, Fonseca MA Maeda L, Taga MSL, Trebachetti CR \& Negrato MLAB (1991). Caracterização físico-química de hidroxiapatita para o uso clínico. In: Zenebon O (Editor), Parecer Técnico a Respeito da Interpretação do Espectro na Região do Infravermelho da Amostra Encaminhada para Análise. Instituto Adolf Lutz, São Paulo.
4. Rubin PAD, Popham JK, Bilk JR \& Shore JW (1994). Comparison of fibrovascular ingrowth into hydroxyapatite and porous polyethylene orbital implants. Ophthalmic Plastic and Reconstructive Surgery, 10: 96-103.

5. Shields CL, Shields JA, Eagle RC \& De Potter P (1991). Histopathologic evidence of fibrovascular ingrowth four weeks after placement of the hydroxyapatite orbital implant. American Journal of Ophthalmology, 111: 363-366. 